\title{
Clinical, Epidemiological and Microbiological Profile of Dengue Fever at a Tertiary Care Hospital in Delhi, India
}

\author{
Avinash Kumar, Sharon Rainy Rongpharpi, Shalini Dewan Duggal, Renu Gur, Sanjay Choudhary and Pratima Khare
}

Department of Microbiology, Dr Baba Saheb Ambedkar Hospital, Rohini, Delhi, India

*Corresponding author: Gur R, Senior Specialist and Head, Department of Microbiology, Dr Baba Saheb Ambedkar Hospital, Rohini, Delhi, India, Tel: +919968679770; E-mail: dr.renugur@yahoo.com; renugur@hotmail.com

Received date: July 09, 2017; Accepted date: August 05, 2017; Published date: August 12, 2017

Copyright: (C) 2017 Kumar A, et al. This is an open-access article distributed under the terms of the Creative Commons Attribution License; which permits unrestricted use; distribution; and reproduction in any medium; provided the original author and source are credited.

\begin{abstract}
Dengue Fever (DF), Dengue hemorrhagic fever (DHF) and Dengue Shock Syndrome (DSS) are topmost public health concerns today, especially in tropical and subtropical countries, mainly involving urban and semi-urban areas. It is estimated that two fifth of the population in tropical countries, around 2.5 billion people are vulnerable. Approximately, 50 million dengue infections occur worldwide of which 500,000 people are hospitalized with DHF annually. Nearly 90 percent of them are children less than five years old, and about 2.5 percent die. Dengue epidemics are occurring at an increased frequency and one or more types of serotypes circulate. During these epidemics, infection rate among virus naïve patients ranges from 40-90 percent. In India, Dengue is hyperendemic (Category A) and is a notifiable infectious disease. Our hospital has been identified as a Sentinel Surveillance centre for diagnosis and treatment of Dengue in North West district of Delhi. Though gaps exist in terms of public health measures and health education among public, microbiological laboratory plays a crucial role in confirmation of dengue infection and estimation of burden of disease.
\end{abstract}

Keywords: Dengue fever; Epidemiology; Clinical; Public health; Infections

\section{Introduction}

Dengue Fever (DF), Dengue hemorrhagic fever (DHF) and Dengue Shock Syndrome (DSS) are topmost public health concerns today, especially in tropical and subtropical countries, mainly involving urban and semi-urban areas. It is estimated that two fifth of the population in tropical countries, around 2.5 billion people are vulnerable. Approximately, 50 million dengue infections occur worldwide of which 500,000 people are hospitalized with DHF annually. Nearly 90 percent of them are children less than five years old, and about 2.5 percent die. Dengue epidemics are occurring at an increased frequency and one or more types of serotypes circulate. During these epidemics, infection rate among virus naïve patients ranges from $40-90$ percent [1]. In India, Dengue is hyperendemic (Category A) and is a notifiable infectious disease [2]. Our hospital has been identified as a Sentinel Surveillance centre for diagnosis and treatment of Dengue in North West district of Delhi. Though gaps exist in terms of public health measures and health education among public, microbiological laboratory plays a crucial role in confirmation of dengue infection and estimation of burden of disease.

\section{Materials and Methods}

This was a prospective study conducted from July 2013 to December 2013 at a referral hospital in North Delhi. Case inclusion criteria included presentation of sudden high fever $\left(39-40^{\circ} \mathrm{C}\right)$ of 2-7 days duration, intense headache, myalgia, arthralgia or rash. The case was excluded if there was evidence of bacterial or other viral illness based on laboratory testing, duplicate samples were excluded. All cases were managed according to WHO Guidelines [3]. The patients with warning signs were transferred to Dengue ward for observation and further management.

After consent of the patient, blood was taken by venipuncture according to standard guidelines. Serum was refrigerated $\left(2-8^{\circ} \mathrm{C}\right)$ if not tested within two days. Sera showing haemolysis, icterus, lipaemia or microbial growth were excluded because they may cause false positive/ negative interpretation. Before beginning assay, all reagents were brought to room temperature $\left(20-25^{\circ} \mathrm{C}\right)$.

Depending upon duration of fever, NS1Antigen or IgM Capture ELISA (Mac ELISA) was done in the Department of Microbiology. Samples obtained in initial phase (within 5 days of sickness) were qualitatively tested for dengue viral NS1 antigen using Panbio Dengue Early ELISA (NS1 antigen capture ELISA). In this kit, wells were coated with purified dengue virus type 2 antigen cultured in Vero cells. The test was executed according to the manufacturer's instructions. Dengue NS1 antigen, if present in serum, binds to anti-NS1 antibodies attached to the polystyrene surface of the micro-wells which is followed by chromogenic reaction. Development of colour was indicative of the presence of dengue NS1 antigen in the test sample. An active primary dengue infection was considered when positive result came (>11 Panbio Units).

Samples of patients with fever of more than five days duration were tested for the presence of anti-dengue IgM antibodies using MAC ELISA NIV (National Institute of Virology), Pune and as recommended by National Vector Borne Disease Control (NVBDCP, Delhi). IgM antibodies in the patient's blood was captured by Antihuman IgM ( $\mu$ chain specific) coated on the wells. In the next step, DEN antigen was added which could capture IgM, if IgM and antigen were homologous. Unbound antigen was removed during the washing step. In the subsequent step Biotinylated flavivirus cross-reactive monoclonal antibody (Hx-B) was added followed by Avidin -HRP. Subsequently, substrate/chromogen was added and noted for 
development of colour. The reaction was stopped by $1 \mathrm{~N} \mathrm{H}_{2} \mathrm{SO}_{4}$. Intensity of colour/optical density was monitored at $450 \mathrm{~nm}$. For quality control, each kit had a positive and a negative control. Calculations and interpretation were done as per the kit literature.

Daily online reports of the serologically positive cases were sent to the Municipal Corporation of Delhi (MCD), from our hospital. Also the weekly data of dengue serological tests were sent in the IDSP (Infectious Diseases Surveillance Project) format to Medical Records Department. Monthly report was also sent to NCDC (National Centre for Disease Control).

\section{Results}

\section{Laboratory profile}

A total of 2205 patients of suspected Dengue presented during the study period. Based on the number of days of fever, 1792 serum samples were tested for anti-dengue IgM antibodies and 413 for NS1 antigen. Out of these, 911 were serologically positive; 699 (39\%) for anti-dengue IgM antibodies and, 212 (51\%) for NS1 antigen (Figure 1). A total of 911 samples tested positive. Maximum number of dengue positive serum samples was observed in the month of October (Figure 2). To look for platelet and leucocyte counts, blood samples were drawn for routine hemogram at the time of admission. This was evaluated by fully automated haematology analyser- 5 part differential (Nihon Kohdon). Thrombocytopenia was defined as platelet counts less than $1,50,000 /$ microliter and confirmed on peripheral smear examination (Figure 3). Leucocyte counts of dengue patients were recorded and in majority of patients they were in normal range (4000-11000 per microliter) (Figure 4).

\section{Clinical profile of dengue}

Out of 911 positive dengue cases, 661 were adult and 250 were of pediatric age group. The lowest age of positive case was 3 months and highest was 70 years. Two hundred and fifty one (27.5\%) dengue samples were positive among patients $<12$ years of age. Male Female ratio was observed to be 1.85 . The Clinical profile of adult and Pediatric Dengue patients was compared in terms of the grading and disease progression.

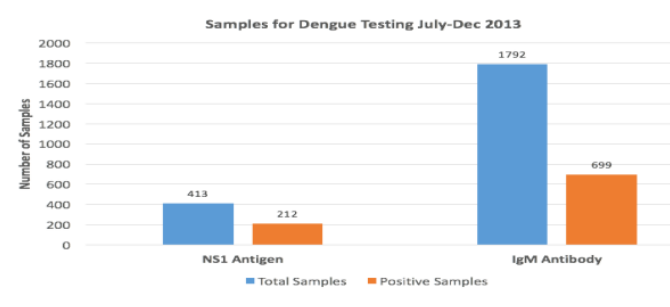

Figure 1: Samples for dengue testing July-Dec 2013.

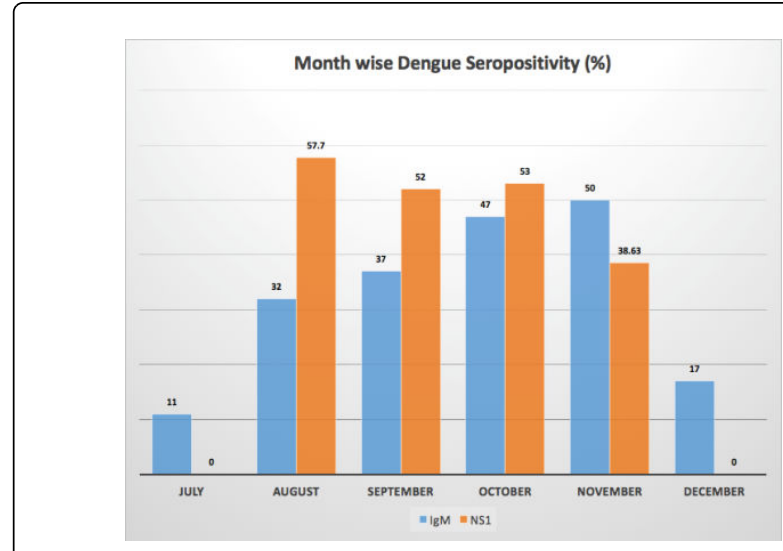

Figure 2: Month wise dengue seropositivity (\%).

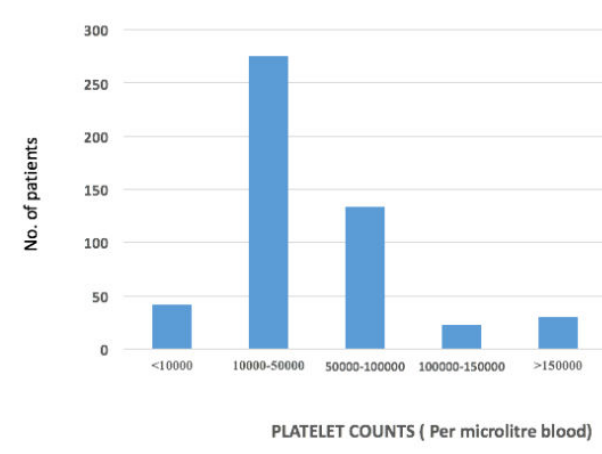

Figure 3: Platelet count (microL).

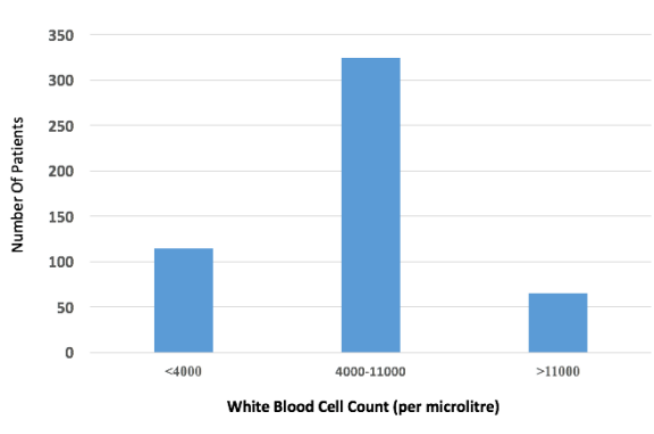

Figure 4: White blood cells count (microL).

Among adults, a majority of dengue patients progressed to DHF II while in pediatric age group most of them progressed to DHF III (Figure 5). Of the Pediatric DHF IV patients, total six children developed complications including myocarditis (2), encephalitis (2), Acute Respiratory Distress Syndrome (1) and pulmonary oedema (1). However, no mortality was observed during the study period, all patients recovered. 


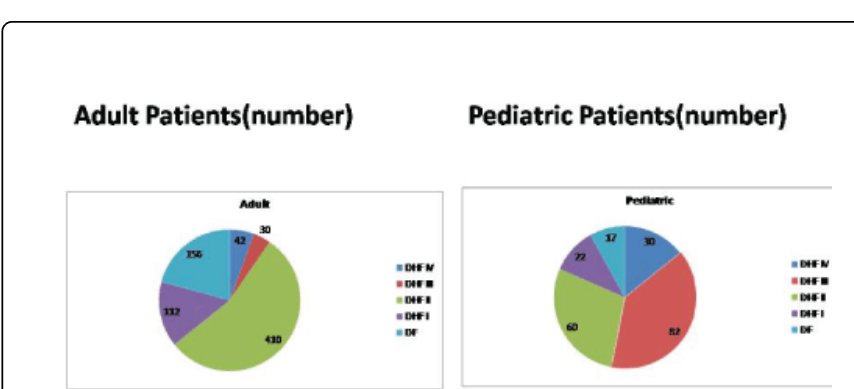

Figure 5: Clinical profile of dengue.

\section{Epidemiological profile of dengue}

Maximum Dengue cases were observed in Rohini Zone (65\%) followed by Narela (20\%), civil lines (6\%) and Central (4\%) zones. Most of the cases were from Budh Vihar and Shahbad Dairy in Rohini zone (Table 1). The doctors, nursing and paramedical staff of our hospital constituted $1.4 \%$ of positive cases. In Narela zone, Nangloi ward had maximum cases while in Civil line Zone most of the cases were from Burari ward (Figure 6). Five percent of our positive cases were from neighbouring states including Haryana, Uttar Pradesh and Bihar of which Sonepat (Haryana) had maximum dengue positive cases probably because of its closer proximity to Delhi.

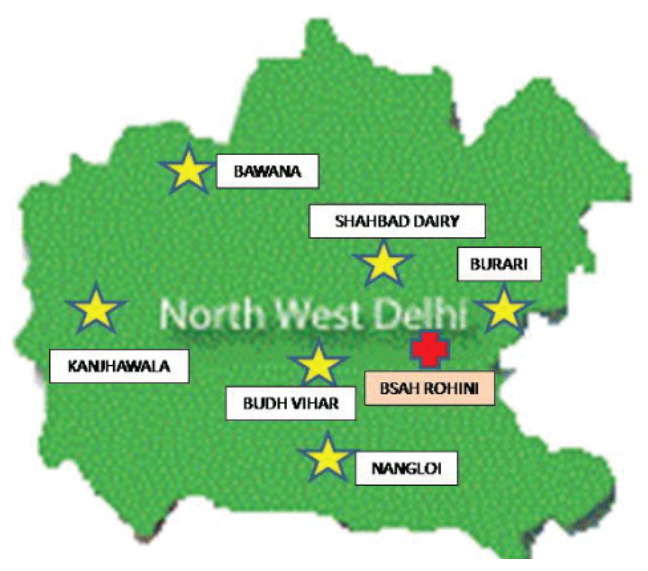

Figure 6: Geographical profile of dengue in north-west Delhi.

\begin{tabular}{|l|l|}
\hline Geographical Area & Cases \\
\hline Rohini Zone & 592 \\
\hline Narela Zone & 182 \\
\hline Civil Zone & 55 \\
\hline Central Zone & 36 \\
\hline Near Capital Territory Zone & 46 \\
\hline
\end{tabular}

Table 1: Demographics of dengue positive population involved in study.

\section{Discussion}

Dengue viruses are arboviruses capable of infecting humans and causing outbreaks. Dengue infection can be caused by any one or more of the four different but closely related serotypes; DEN1, DEN2, DEN3 or DEN4 dengue virus of the genus Flavivirus [1]. Dengue fever (DF) is a self-limiting disease in majority of cases, rarely it may cause Dengue Hemorrhagic Fever (DHF) and Dengue Shock Syndrome (DSS) [3]. DF has been defined as fever with two or more symptoms including headache, retro-orbital pain, myalgia or arthralgia and leucopenia, thrombocytopenia. There are four grades of severity of DHF. DHF -I include above criteria for DF plus positive tourniquet test, evidence of plasma leakage with platelet count less than 100,000/ cu.mm and haematocrit rise $20 \%$ or more. DHF II includes DHF I plus some evidence of spontaneous bleeding in skin or other organs (black tarry stools, epistaxis, bleeding from gums, etc.) and abdominal pain. DHF III includes DHF II plus circulating failure (weak rapid pulse, pulse pressure $<20 \mathrm{~mm} \mathrm{Hg}$ or high diastolic pressure, hypotension with the presence of cold clammy skin and restlessness). DHF IV incudes profound shock with undetectable blood pressure or pulse and haematocrit rise more than $20 \%$. DHF III and IV are the criteria for labelling as DSS [3].

Early diagnosis may be challenging as dengue infection presents with non-specific signs and symptoms which may not be easily differentiated from other febrile illnesses. In our study, peak incidence of dengue occurred in the month of October which correlates with other Indian studies $[4,5]$. This may be supported by scientific evidence of temperature and rainfall influencing dengue incidence. According to IDSP data, dengue fever cases peak during the post monsoon period between mid-September to November [6]. The density of Aedes aegypti in an area is also linked with rainfall and water storage. Life span of this vector is influenced by temperature and humidity. It survives best between $16^{\circ} \mathrm{C}-30^{\circ} \mathrm{C}$ and a relative humidity of $60-80 \%$; breeds in containers in and around the house. It has been observed that even a $2^{\circ} \mathrm{C}$ rise in temperature can reduce the extrinsic incubation period of DENV, hence an increase in mosquito density. The extent of dengue transmission depends on interplay of multiple factors including host population density, vector density and proportion of non-immunized people in a community [1-7].

Dengue fever has assumed an endemic status in more than 100 countries in Africa, America, Eastern Mediterranean, South-East Asia and Western Pacific areas. South-East Asia and Western Pacific regions are worst affected. Climatic variation spread of dengue vectors to newer geographic regions, increasing cross border travel, global trade, and urban movement have collectively raised the status of dengue fever to a problem of global concern [8]. In India, the vulnerability of dengue has increased in recent years due to rapid urbanization, lifestyle changes and deficient water management including improper water storage practices in urban, periurban and rural areas, leading to proliferation of mosquito breeding sites. Most of the cases are from unauthorized dwellings where improper drainage of water was present. Lack of potable water, poor hygienic conditions, overcrowding, and stored water may lead to potential breeding sites favourable for the mosquitoes However, in the Southern states and Gujarat the transmission is perennial [9].

Dengue is endemic in 31 States/UTs. During 2011, about 18,059 cases where reported with 119 deaths and the case fatality rate was 0.65 percent. The highest numbers of cases were reported from Punjab followed by Tamil Nadu, Gujarat, Kerala, and Andhra Pradesh [9]. All four serotypes i.e., dengue 1,2,3,4 have been isolated in India [3]. In 
Delhi, DENV-3 strain became predominant from 2003-2006. After that, DENV-1 became predominant and one or more of these serotypes concomitantly circulate in the absence of cross immunity [10].

Over the past few decades, there has been an enormous increase in the frequency of dengue epidemics. Epidemics from India include those from Calcutta (1963), Vishkapattanam (1964), Asansole of West Bengal, Vellore (1968), Ajmer (1969), Kanpur (1969), Delhi (1970), Jalore of Rajasthan (1985) and from Delhi in 1996 [11]. Delhi now comes under hyperendemic zone for dengue, the last major outbreak reported in 2006 [12]. In Delhi, dengue cases rose rapidly with 2,092 cases including 3 deaths reported until September 2013 compared to 65 cases and 3 deaths reported during a corresponding period in 2012. The worst affected areas in 2013 had been Rohini, Narela, Najafgarh, Shahdara (North), Karol Bagh and central Delhi zones [6]. Aedes aegypti was identified as the only vector in these areas though $A$. albopictus is another important dengue vector. Aedes aegypti is strongly anthrophilic, a nervous feeder (bites more than one host to complete a blood meal) and a discordant species (needs more than one feed for completion of its gonotropic cycle). In contrast, Aedes albopictus is an aggressive feeder and concordant species.

In dengue, patient generally complains of abrupt onset of high fever accompanied by severe frontal headache, retro-orbital pain which worsens with eye movements, joint and muscular pains, loss of taste and appetite. Measles-like rash may appear in 80 percent of cases over chest and upper limbs indicating haemorrhages under the skin. Males are more frequently exposed to the risk of acquiring dengue than females because of their outdoor activity, especially working near construction sites, clogged drains, and accumulated wastes [13]. In our study; Male-to-female ratio was $1.85: 1$ which is comparable with the studies in Delhi [14].

Diagnosis of dengue infection is confirmed by the detection of virus, viral genome or NS1 Antigen, or seroconversion of IgM or IgG (from negative to positive IgM/IgG or four-fold increase in the specific antibody titre) in paired sera. Of all the methods available for dengue diagnosis, virus isolation provides the most specific test result. However, facilities that can support viral culture are not always available. The detection of the viral genome or viral antigens also provides evidence of infection. A large window of opportunity for Dengue diagnosis is provided by NS1 Ag which is highly conserved glycoprotein. Single IgM ELISA test positivity is probable of dengue and definitive diagnosis requires use of paired sera to demonstrate rising titre [15].

Infection with any serotype confers lifelong immunity to that virus serotype. Even though all four serotypes are antigenically similar, they can elicit cross protection for only a few months after infection. Secondary infection with dengue serotype 2 or multiple infections with different serotypes lead to severe form of dengue DHF/DSS [1]. The first infection probably sensitizes the patient, while the second infection with different serotype appears to produce immunologically enhanced reaction. The 2006 outbreak in Delhi was due to multiple circulating serotypes, Den-3 being the most common circulating serotype [16].

In India, 527 sentinel surveillance centres, including ours and 14 apex referral laboratories have been established [17]. Management of dengue primarily includes symptomatic and supportive care. No effective anti-viral drugs yet exist to treat the illness. Encouragement of taking oral rehydration solution (ORS), fruit juice and other fluids containing electrolytes and sugar. Paracetamol only is to be given for high fever while NSAIDS is contraindicated. In case of DSS, replacement of lost plasma, correction of electrolyte and metabolic disturbances, and a blood transfusion is recommended. Prophylactic platelet transfusion may be given at level of $<10000 /$ cu.mm, prolonged shock; coagulopathies and in systemic massive bleeding [3].

Vector control is the primary means of controlling dengue till vaccines become widely available. Vector control measures include eradication of Aedes mosquito breeding sites; mosquito proofing of overhead and ground tanks, and buildings; use of mosquito nets to prevent cross infection in hospitals; and use of pyrethrum space spray/ULV during outbreaks. Since they feed during the day, insecticide- treated bed nets are also an ineffective intervention to reduce number of bites [1].

This is the first study, which depicts the areas which were highly endemic for dengue cases in North West of Delhi. Most of the cases were from unauthorized dwellings where improper drainage of water was present. Lack of potable water, poor hygienic conditions, overcrowding, and stored water may lead to potential breeding sites favourable for the mosquitoes. After receiving positive dengue reports, the municipal corporation of Delhi inspected the residential area of patient and fogging measures initiated to prevent further spread of the disease. Also the municipal authorities had been authorized to charge penalty from the concerned individual household. Training and Lectures were conducted in our hospital regarding management and diagnosis during dengue epidemics.

The study also reveals that dengue infection is also prevalent in adjacent areas of Delhi like Sonepat district of Haryana, Uttar Pradesh and Bihar. This suggests that dengue infection is no more an urban area infection but it is extending its arms to rural areas also, which may become a cause for concern to health authorities. This study calls for a careful vigil to prevent the possible spread of the vectors between different areas.

The overall cost of illness includes direct medical cost, direct nonmedical costs (e.g., travel) and indirect costs (the value of time lost from morbidity and premature death) [18]. In low- and middleincome countries, direct medical costs have been $66 \%$ of overall economic costs for hospitalized episodes and $23 \%$ of overall costs for ambulatory episodes [19]. Therefore, overall annual economic costs of dengue illness in India would be about $\$ 1.11$ billion or $\$ 0.88 /$ capita and medical cost per day of illness is about $\$ 6.77$ ( $\$ 94.85 / 14$ days) [20]. In our study, financial burden of dengue per patient can be estimated to be around Rs.20,000-25,000, which included cost of testing, diagnosis, management, and testing of blood components if required.

\section{Limitations}

Molecular testing to know the serotype of dengue virus could not be done.

\section{Conclusion}

Dengue has become widespread and repeated attacks due to different serotypes are becoming common. Early diagnosis of dengue allows institution of appropriate supportive therapy and decreases risk of complications. In this regard, the role of the laboratory for diagnosis of Dengue, through serological, molecular and virological methods remains crucial to understand the exact burden of disease. Monitoring of climatic and environmental factors has important association with 
Citation: Kumar A, Rongpharpi S, Duggal SD, Gur R,Choudhary S, et al. (2017) Clinical, Epidemiological and Microbiological Profile of Dengue

Page 5 of 5

high vector density. Understanding of local epidemiology, risk factors and disease burden in dengue is essential to initiate preventive measures in time so that the outbreaks and epidemics can be avoided or controlled.

\section{Conflict of Interest}

None.

\section{References}

1. WHO (2011) Comprehensive guidelines for prevention and control of dengue and dengue hemorrhagic fever, revised and expanded edition, SEAR.

2. Park K (2013) Preventive and social medicine: Dengue syndrome (22nd edn). Banarasidas Bhanot, Jabalpur.

3. National Guidelines for Clinical Management of Dengue Fever (2015) National Vector Borne Disease Control Programme, World Health Organization, India.

4. Chandy S, Ramanathan K, Manoharan A, Mathai D, Baruah K (2013) Assessing effect of climate on the incidence of dengue in Tamil Nadu. Indian J Med Microbiol 31: 283-286.

5. Neeraja M, Lakshmi V, Teja VD, Umabala P, Subbalakshmi MV (2006) Serodiagnosis of dengue virus infection in patients presenting to a tertiary care hospital. Indian J Med Microbiol 24: 280-282.

6. National Centre for Disease Control Newsletter (2013) Quarterly newsletter from the national centre for disease control (NCDC).

7. Karim MN, Munshi SU, Anwar N, Alam MS (2012) Climatic factors influencing dengue cases in Dhaka city: A model for dengue prediction. Indian J Med Res 136: 32-39.

8. Bhatt S, Gething PW, Brady OJ, Messina JP, Farlow AW, et al. (2013) The global distribution and burden of dengue. Nature 496: 504-507.
9. http://dghs.gov.in/

10. Gupta E, Ballani N (2014) Current perspectives on the spread of dengue in India. Infect Drug Resist 7: 337-342.

11. Sharma RS, Kumari R, Srivastava PK, Barua K, Chauhan LS (2014) Emergence of dengue problem in India-A public health challenge. J Commun Dis 46: 17-45.

12. No Authors Listed (2006) Status note on dengue fever / dengue haemorrhagic fever.

13. Wali JP, Biswas A, Handa R, Aggarwal P, Wig N, et al. (1999) Dengue hemorrhagic fever in adults: a prospective study of 110 cases. Trop Doct 29: 27-30.

14. Gupta E, Dar L, Narang P, Srivastava VK, Broor S (2005) Serodiagnosis of dengue during an outbreak at a tertiary care hospital in Delhi. Indian J Med Res 121: 36-38.

15. Dengue (2009) Guidelines for diagnosis, treatment prevention and control. TDR/WHO. WHO/HTM/NTD/DEN, Geneva.

16. Bharaj P, Chahar HS, Pandey A, Diddi K, Dar L, et al. (2008) Concurrent infections by all four dengue virus serotypes during an outbreak of dengue in 2006 in Delhi, India. Virol J 5:1.

17. No Authors Listed (2016) List of sentinel surveillance hospitals for dengue and chikungunya.

18. Halasa YA, Shepard DS, Zeng W (2012) Economic cost of dengue in Puerto Rico. Am J Trop Med Hyg 86: 745-752.

19. Suaya JA, Shepard DS, Siqueira JB, Martelli CT, Lum LC, et al. (2009) Cost of dengue cases in eight countries in the Americas and Asia: A prospective study. Am J Trop Med Hyg 80: 846-855.

20. Pantoja A, Lonnroth K, Lal SS, Chauhan LS, Uplekar M, et al. (2009) Economic evaluation of public-private mix for tuberculosis care and control, India. Part II. Cost and cost-effectiveness. Int J Tuberc Lung Dis 13: 705-712. 